Objectives: Our study aimed to define chemokine receptor profile of peripheral OCPs in rheumatoid arthritis (RA), with comparison to psoriatic arthritis (PSA), as well as their susceptibility to chemotactic signals.

Methods: 129 RA, 53 PSA and 110 control patients were enrolled after Ethical approval. PB samples and synovial fluid (SF) samples, with clinical data of disease activity, inflammation and autoantibody levels were collected. Patients starting anti-TNF therapy were followed up 6 months. TNF- $\alpha$ and CTX serum levels were measured by ELISA. Frequency of OCP-rich subpopulation (CD3CD19-CD56-CD11b+CD14+), expression of OC differentiation (CD115, RANK) and chemokine (CCR1, CCR2, CCR4, CXCR4) receptors was assessed by flow cytometry. OCPs were sorted using FACS, cultured with M-CSF and RANKL, stained for TRAP enzyme and mature OCs counted. Levels of CCL2, CCL3, CCL4, CCL5, CXCL9 and CXCL10 were measured using cytometric bead array, and of CXCL12 by ELISA. Osteoclastogenic effects of CCL2, CCL5 and CXCL10 were analyzed in cell culture, and chemotactic effects on OCPs were studied by cell migration assay using Transwell, with count of number of migrated cells and subsequently differentiated mature osteoclasts.

Results: OCP population was moderately enlarged in $\mathrm{PB}$, further expanded in SF and correlated with TNF- $\alpha$ and rheumatoid factor (RF) levels in patients with RA. However, sorted OCPs generated similar number of mature OCs as control. RANK + subpopulation was enlarged in SF vs PB and correlated with number of tender joints. In PSA, the OCP population was not enlarged, but had a higher RANK expression. OCPs in RA and PSA had higher expression of CCR1, CCR2, CCR4, CXCR4, and all except CCR4 showed positive PB-to-SF gradient. RA had higher levels of CCL2, CCL3, CCL4, CCL5, CXCL9 and CXCL10, with a positive PB-to-SF gradient for all except CCL5 and CXCL9. OCP frequency correlated with levels of CCL2 and CCL5. Subset expressing CXCR4 was associated with TNF- $\alpha$, CTX and RF levels and was lower in patients treated with DMARD, who at the same time had lower osteoresorption (CTX). Subset expressing CCR4 showed significant negative trend during anti-TNF treatment. CCL2, CCL5 and CXCL10 showed significant osteoclastogenic effect. CCL5 showed greatest chemotactic effect, attracting the highest number of cells in the migration assay. At the same time, attracted cells possessed greater osteoclastogenic potential.

Conclusions: Our study provides evidence of the specific importance of certain chemokine signals for stimulation of OCP mobilization, subsequent tissue homing, and maturation, explaining local as well as generalized bone loss seen in RA. Novel insights in regards to migratory behavior and functional properties of PB OCPs in response to chemotactic signals could open way to new therapeutic targets in RA.

Acknowledgements: This work was supported by a grant from the Croatian Science Foundation (project number 5699).

Disclosure of Interest: None declared

DOI: 10.1136/annrheumdis-2017-eular.2317

\section{AB0027 EXPRESSION DENSITY OF RECEPTORS TO TNF-ALPHA IS ASSOCIATED WITH DAS-28 SCORE IN RHEUMATOID ARTHRITIS}

A.A. Alshevskaya ${ }^{1}$, J. Lopatnikova ${ }^{1}$, N. Shkaruba ${ }^{1}$, O. Chumasova $^{1}$, A. Sizikov ${ }^{1}$, V. Lukinov ${ }^{2}$, A. Moskalev ${ }^{2,3}$, S. Sennikov ${ }^{1} .{ }^{1}$ Federal State Budgetary Scientific Institution "Research Institute of Fundamental and Clinical Immunology"; ${ }^{2}$ EOL Labs Ltd; ${ }^{3}$ Biostatistics and Clinical Trials Center, Novosibirsk, Russian Federation

Background: For a number of cytokines and growth factors density of receptors expression has been shown to be important in regulation of action intensity and variation. Although TNF-alpha is actively involved in the development and progression of chronic inflammation in rheumatoid arthritis (RA) role of membrane receptors and their regulatory function in RA remains unclear.

Objectives: To assess associations of membrane-bound receptors expression and soluble receptors and cytokine content with disease activity score in patients with rheumatoid arthritis.

Methods: To reveal linear relationships among integrated score DAS-28 and studied parameters of intact T-cells, B-cells and monocytes (evaluated by flow cytometry) and parameters of mediators soluble content (evaluated by ELISA) building of multiple linear regression model (MLRM) with a standard assessment of the regression coefficients by least squares method were used. To determine receptor number on the cells QuantibritePE Beads (BD) were used.

Results: For the group of patients with acute stage of RA MLRMs with best statistical quality characteristics revealed that for lymphocytes both TNFR1 and TNFR2 number had significant associations with DAS-28; for monocytes only number of TNFR2 was significant. Lower DAS-28 index is associated with higher number of TNFR2 per cell and lower number of TNFR1.

Comparison of models with different combinations of studied TNFR-parameters revealed that percentages of cells expressing receptors for TNF $\alpha$ validated not more than $20 \%$ of DAS- 28 variation, while the number of receptors indicators on cells validated $40-50 \%$ of DAS-28 variation. Full MLRM with all 15 studied parameters validated about $70 \%$ of DAS- 28 variation.

For the group of patients with acute stage of RA MLRMs with best statistical quality characteristics revealed that for lymphocytes both TNFR1 and TNFR2 number had significant associations with DAS-28; for monocytes only number of TNFR2 was significant. Lower DAS-28 index is associated with higher number of TNFR2 per cell and lower number of TNFR1. Comparison of models with different combinations of studied TNFR-parameters revealed that percentages of cells expressing receptors for TNF $\alpha$ validated not more than $20 \%$ of DAS-28 variation, while the number of receptors indicators on cells validated $40-50 \%$ of DAS-28 variation. Full MLRM with all 15 studied parameters validated about $70 \%$ of DAS-28 variation.

Conclusions: Number of receptors to TNF-alpha is more associated with RA activity score as compared with soluble receptors or cytokine. Number of receptors type 1 and type 2 to TNF-alpha per cell have opposite influence on disease activity score. Our findings indicate the involvement of receptors expression density changes in the pathological process in RA.

Disclosure of Interest: None declared

DOI: 10.1136/annrheumdis-2017-eular.2643

\section{AB0028 FIBROBLAST-LIKE SYNOVIOCYTES MAY NOT BE THE TARGET OF IL-33 IN THE JOINT PHISIOPATHOLOGY}

C.R.L. Machado ${ }^{1}$, G.G. Resende ${ }^{2}$, R.B.V. Macedo ${ }^{2}$, V.C. Nascimento ${ }^{2}$, T.P. Silva ${ }^{2}$, A.M. Kakehasi ${ }^{2}$, M.V.M. Andrade ${ }^{2} .{ }^{1}$ Universidade Federal Minas Gerais: ${ }^{2}$ Universidade Federal de Minas Gerais, Belo Horizonte, Brazil

Background: Rheumatoid arthritis (RA) and osteoarthritis (OA) are chronic joint diseases in which fibroblast-like synoviocytes (FLS) actively participate in the synovitis- damage cycle, trough production of cytokines such as IL-6 and metalloproteinases $(\mathrm{MMPs})^{(1)}$. It has already been showed that serum IL-33 levels correlated with disease activity in RA ${ }^{(2)}$. IL-33 is capable to enhance TNF- $\alpha$ effects in $\mathrm{FLS}^{(3)}$. In the collagen-induced arthritis (CIA) model, the injection of IL-33 exacerbated the disease ${ }^{(4)}$. Since ST2 receptor is expressed in FLS, it is hypothesized that IL-33 could activate FLS and increase downstream production of inflammatory cytokines.

Objectives: To evaluate the production of IL-6, MMP-1, and MMP-3 by FLS stimulated with IL-33, TNF- $\alpha$, and IL-1 $\beta$.

Methods: FLS were cultured from samples of synovial fluid and tissue of $O A$ patients (OAFLS $n=8$ ), RA patients (RAFLS $n=3$ ), and patients without rheumatic disease (health) (HFLS $n=4$ ). FLS were stimulate with TNF- $\alpha$ at concentrations of $1 ; 5 ; 10$ and $50 \mathrm{ng} / \mathrm{ml}$, IL-1 $\beta$ at concentrations of $0.1 ; 0.2 ; 0.3 ; 0.5$ and $1 \mathrm{ng} / \mathrm{ml}$ and IL-33 at concentrations of $1 ; 3 ; 10 ; 30 ; 100 \mathrm{ng} / \mathrm{ml}$, soon after, IL-6, MMP-1, and MMP- 3 levels were evaluated by ELISA, in the cell supernatant.

Results: MMP-1, MMP-3 and IL-6 were constitutively expressed by FLS at baseline in all groups. Both TNF- $\alpha$ and IL- $1 \beta$ stimulated the production of IL-6 and MMP-1 with statistical significance in a dose-dependent manner in all three groups. Only IL- $1 \beta$ increased the production of MMP-3. TNF- $\alpha$ stimulated the production of MMP-3 only on HFLS. There was no difference between the concentration of MMP-1, MMP-3 and IL6 in the supernatant of OAFLS, ARFLS and HFLS when IL-33 stimulated and non-stimulated were compared.

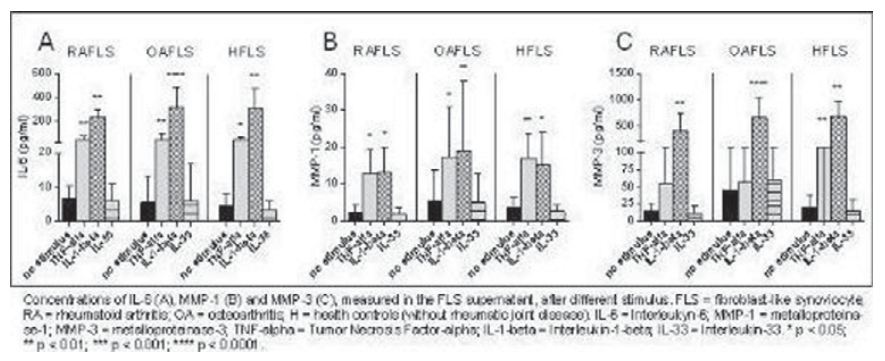

Conclusions: This study demonstrated that IL-33 failed to induce the production of IL-6, MMP- 1 and MMP-3 by FLS of different diseases sources, suggesting that must be another cell type that plays the role of target to IL-33 in physiopathology of joint inflammation. The absence MMP-3 in response to TNF- $\alpha$ stimulus in RAFLS and OAFLS could be explain by saturation of this cytokine in synovial cells from these diseases.

References:

[1] Bartok B, Firestein GS. Fibroblast-like synoviocytes: key effector cells in rheumatoid arthritis. Immunological reviews. 2010;233(1):233-55.

[2] Matsuyama Y, Okazaki H, Tamemoto H, Kimura H, Kamata Y, Nagatani K, et al. Increased levels of interleukin 33 in sera and synovial fluid from patients with active rheumatoid arthritis. The Journal of rheumatology. 2010;37(1):18-25.

[3] Kunisch E, Chakilam S, Gandesiri M, Kinne RW. IL-33 regulates TNF-alpha dependent effects in synovial fibroblasts. International journal of molecular medicine. 2012;29(4):530-40.

[4] Xu D, Jiang HR, Kewin P, Li Y, Mu R, Fraser AR, et al. IL-33 exacerbates antigen-induced arthritis by activating mast cells. Proceedings of the National Academy of Sciences of the United States of America. 2008;105(31):10913-8.

Acknowledgements: Capes, Fapemig and Fundo de Apoio a Pesquisa e Ensino da Sociedade Brasileira de Reumatologia - FAPE-SBR.

Disclosure of Interest: None declared

DOI: 10.1136/annrheumdis-2017-eular.6673 